\title{
Clinico-Mycological study of tinea pedis and toe nail onychomycosis: A multi-center study from Jammu
}

\author{
Ramjan Bhatti ${ }^{1}$, Parvaiz A Rather ${ }^{2, *}$, Devraj Dogra ${ }^{3}$, Vipin Gupta ${ }^{4}$, Bella Mahajan ${ }^{5}$ \\ ${ }^{1}$ Dermatologist, ${ }^{2}$ Assistant Professor,,${ }^{3,5}$ Professor and Head, ${ }^{4}$ Ex. Professor, ${ }^{1-4}$ Dept. of Dermatology, ${ }^{5}$ Dept. of Microbiology, ${ }^{2-}$ \\ ${ }^{5}$ Government Medical College, Jammu, District Hospital Rajouri, Jammu and Kashmir, India
}

*Corresponding: Parvaiz A Rather

Email: parvaizanwar@gmail.com

\begin{abstract}
Introduction: Tinea Pedis (T. Pedis) and toe nail Onychomycosis is a chronic relapsing superficial fungal infection, often associated with that of other cutaneous areas. Dermatophytes are the commonest organisms in T. Pedis and Onychomycosis, Trichophyton Rubrum (T. Rubrum) being the commonest isolate. The causative species may be different from place to place, so it is important to have a knowled ge about the dermatophytes causing T. Pedis and Onychomycosis in a particular area, so that specific agents are used for effective treatment, and prevent drug resistance.

Aims: Study morphological patterns of T. Pedis and toe nail Onychomycosis and find causative fungal species.

Material and Methods: Prospective hospital based study over 1 year period, on clinically diagnosed cases of T. Pedis and toe nail Onychomycosis, confirmed by positive results on microscopic examination of $\mathrm{KOH}$ preparation. Culture was done to isolate the specific pathogen involved.

Results: Out of 150 patients, 60 (40\%) had T. Pedis alone, $34(22.7 \%)$ Toe nail Onychomycosis alone and 56 (37.3\%) both T. Pedis and Onychomycosis in the same patient. On culture, 56 patients out of $150(37.3 \%)$ showed a positive fungal growth. 30 out of 56 patients $(53.3 \%)$ of mixed T. Pedis and Onychomycosis were positive. 16 out of 60 patients (26.7\%) of T. Pedis and 10 out of 34 (29.4\%) Onychomycosis patients respectively were culture positive.

Conclusion: Among all the clinically diagnosed and $\mathrm{KOH}$ positive patients, culture positivity was established in $56(37.3 \%)$ patients only, T. Rubrum being the most common fungal strain, followed by T. Mentagrophyte.
\end{abstract}

Keywords: Tinea pedis, Onychomycosis, Fungal culture, Trichophyton rubrum, Trichophyton mentagrophyte.

\section{Introduction}

Tinea Pedis is a common relapsing superficial fungal infection of the feet, affecting $10-15 \%$ of population worldwide, with four main clinical variants which include chronic intertriginous type (also known as 'Athlete's foot'); chronic papulosquamous type; vesicular/ vesicobullous type and acute ulcerative type. ${ }^{1}$ Most common causative species of $\mathrm{T}$. Pedis have been found to be anthropophilic dermatophytes especially Trichophyton rubrum, Trichophyton mentagrophtes and Epidermophyton floccosum. ${ }^{2}$ Trichophyton tansurans has also been implicated in children.

Onychomycosis denotes fungal infection of nail plate of toes caused by different species of dermatophytes, yeasts and non-dermatophyte fungi and is one of the commonest nail disorders, accounting for about $30 \%$ of superficial fungal infections of skin. Main clinical patterns of dermatophytic Onychomycosis are Distal or lateral subungual Onychomycosis (DLSO); Total dystrophic subungual Onychomycosis (TDO); Proximal Subungual Onychomycosis (PSO); Superficial white Onychomycosis (SWO). ${ }^{3-6}$ DLSO is the most common pattern. PSO is the least common pattern seen predominantly in immune compromised patients such as those with HIV and AIDS.,

Trichophytons and Epidermophytons are the common species of dermatophytes affecting toe nails. In addition, a number of various opportunistic non-dermatophyte moulds colonise the feet and nails primarily damaged by trauma or some other dermatoses. ${ }^{8,9}$
The prevalence of Tinea Pedis and Onychomycosis of toes are determined by various predisposing factors, such as age, socio-economic status; occupation; climate; living condition; sharing of bathing facilities at sports clubs and swimming pools; wearing of occlusive shoes and clothing; frequent travels and population shifts, along with immunosuppression, local trauma and impaired circulation.

The clinical diagnosis of Tinea Pedis and Onychomycosis can be confirmed by direct microscopy of potassium hydroxide $(\mathrm{KOH})$ preparation. Fungal culture is necessary to identify the specific genus and species of pathogen, as clinical appearance caused by one species of fungus is indistinguishable from that caused by any other species. ${ }^{10}$ Sabouraud's dextrose agar being one of the most commonly used mediums.

The etiological role of a particular dermatophyte in Tinea Pedis and Onychomycosis depends upon its ecological distribution and infection by the same pathogen at any other body site. If a particular dermatophyte is prevalent in a given area it is likely to be the most common cause of fungal infection in that area. The causative species may be different from place to place. So it is important to have a knowledge about the dermatophytes causing Tinea Pedis and Onychomycosis in a particular area, so that specific agents are used for effective treatment and prevent drug resistance.

We undertook this study at two centres simultaneously, a tertiary centre and a secondary referral centre, to evaluate 
the patterns of Tinea Pedis and toe nail Onychomycosis, and find out the causative organisms in this part of the World.

\section{Material and Methods}

It was a prospective hospital based clinical study carried out over a period of 1 year at 2 centres simultaneously, one a tertiary centre and other a secondary referral centre. Clinically suspected patients of Tinea Pedis and toe nail Onychomycosis, microscopically proven to be positive for fungal elements were taken up for study after proper informed consent. Patients with history of any oral medication for any skin diseases for last 3 months and topical application for last 1 month were excluded.

Besides collection of demographic data, a detailed history of every patient was recorded in a format particularly with reference to duration of illness, occupation, hobbies and recreational activities, type of foot wear, contact with pets and cattle, fungal infection elsewhere on the body, presence of fungal infection in other family members, significant drug intake and co-morbid condition.

In addition to general physical and systemic examination, a detailed mucocutaneous examination of feet, toe nails and rest of the body was conducted by the same dermatologists and recorded in the format.

Specimen collection for microscopic examination of $\mathrm{KOH}$ preparation as well as for fungal culture was done as per the standard procedure.
Only $\mathrm{KOH}$ positive sample were inoculated into two to three sets of culture media, such as Sabourand's dextrose agar (SDA) with chloramphenicol; SDA with chloramphenicol and cycloheximide; Dermatophyte test medium (DTM). Each set of media was incubated at room temperature and $37^{\circ} \mathrm{c}$ and examined for any growth of fungi for 4 weeks. Minimal culture time to call it negative was 21 days.

Statistical analysis of the data was done with the help of computer software M S Excel of windows. Descriptive characteristics have been presented with the help of summary measures like mean; standard deviation and qualitative variable have been presented as percentages.

\section{Results}

The study was conducted on 150 prospective patients of T.Pedis and toe nail Onychomycosis, who visited the outpatient department of our respective hospitals.

In this study, Patients ranged from 2-71 years of age with mean age at presentation 35.4 years. Most of the patients were in the age group 21-40 years followed by 4160 years, and males outnumbered females. Various demographic and other variables are presented in summarized form in table 1 .

Table 1: various demographic and other variables of the.

\begin{tabular}{|c|c|c|c|}
\hline Variable & Males & Females & Number \& (\%) \\
\hline Age group (years) & 12 & 4 & $16(10.9)$ \\
\hline$\leq 20$ & 74 & 22 & $96(64.3)$ \\
\hline $21-40$ & 24 & 10 & $34(22)$ \\
\hline $41-60$ & 4 & 0 & $4(2.8)$ \\
\hline$\geq 61$ & $114(76)$ & $36(24)$ & 150 \\
\hline Total & Rural & & $87(58)$ \\
\hline Residential status & Urban & & $63(42)$ \\
\hline & Total & & 150 \\
\hline & & & $60(40)$ \\
\hline Type of disease & T. Pedis & & $36(37.3)$ \\
\hline & Onychomycosis & & \\
\hline & & & $20(62.5)$ \\
\hline Coexisting fungal infection & T. Cruris & & $6(18.8)$ \\
\hline & T. Corporis & & $2(6.2)$ \\
\hline & T. Manum & & $32.7)$ \\
\hline & T. Cruris \& T. Manum & & $32.5)$ \\
\hline
\end{tabular}

Associated comorbid systemic diseases were found in 10 patients, 5 with diabetes mellitus and hypertension in 5 , already on treatment and under control.
Various morphological patterns of T.Pedis and toe nail Onychomycosis are shown in table 2. 
Table 2: Morphological patterns of T. Pedis and toe nail Onychomycosis.

\begin{tabular}{|c|c|c|c|}
\hline T. Pedis & No Of patients & Onychomycosis & No Of patients \\
\hline Intertriginous type & $54(46.5)$ & DLSO & $45(50)$ \\
\hline Squamous hyperkerototic type & $51(44)$ & TDO & $22(24.4)$ \\
\hline $\begin{array}{l}\text { Intertriginous \& Squamous } \\
\text { hyperkerototic type }\end{array}$ & $9(7.8)$ & DLSO \& TDO & $18(20)$ \\
\hline \multirow[t]{3}{*}{ Vesico or vesicobullous types } & $2(1.7)$ & SWO & $3(3.3)$ \\
\hline & & DLSO \& SWO & $2(2.2)$ \\
\hline & & $\mathrm{PSO}$ & 0 \\
\hline Total & 116 & & 90 \\
\hline
\end{tabular}

Out of all the $150 \mathrm{KOH}$ positive patients, 56 patients (37.3\%) showed a positive fungal growth on culture. Among 56 patients of mixed Tinea Pedis and Onychomycosis, 30 (53.6\%) were positive. Likewise among
60 patients of isolated T. Pedis and 34 with Onychomycosis, $16(26.7 \%)$ and $10(29.4 \%)$ respectively showed positive culture results. The results are summarized in table 3 .

Table 3: Isolation of fungal strains on culture of the patient samples

\begin{tabular}{|l|c|c|c|}
\hline \multicolumn{1}{|c|}{ Type of infection } & No. of patients (n=150) & No. of patients positive on SDA & Percentage \\
\hline $\begin{array}{l}\text { Tinea Pedis and } \\
\text { Onychomycosis }\end{array}$ & 56 & $24+6^{*}=30$ & $53.6 \%$ \\
\hline Tinea Pedis & 60 & $14+2^{*}=16$ & $26.7 \%$ \\
\hline Onychomycosis & 34 & $8+2^{*}=10$ & $29.4 \%$ \\
\hline \multicolumn{2}{|c|}{ Total } & $\mathbf{5 6}$ & $\mathbf{3 7 . 3 \%}$ \\
\hline *non dermatophyte isolates on culture media & \\
\hline
\end{tabular}

T. Rubrum alone was isolated in 28 positive culture results and associated with A. Niger in 2 isolates. Out of these, 14 culture isolates had mixed T.Pedis and Onychomycosis, 10 had T.Pedis only and 4 had Onychomycosis only.

T. Mentagrophytes was isolated alone in 18 culture results and in association with A. Niger in 2. Out of these,
10 culture isolates had mixed T.Pedis and Onychomycosis, and 4 each had T.Pedis and Onychomycosis alone.

Growth of A. Niger and Candida albicans in isolation was found in 2 cases each. This is summarized in table 4 .

Table 4: Distribution of fungal species in culture positive patients.

\begin{tabular}{|l|c|c|c|c|c|}
\hline \multicolumn{1}{|c|}{ Isolate } & TP \& O* & TP* & O* & Total & Percentage \\
\hline T. Rubrum & 14 & 10 & 4 & 28 & $50 \%$ \\
\hline T. Mentagrophyte & 10 & 4 & 4 & 18 & $32.1 \%$ \\
\hline T. Rubrum \& A. Niger & - & 2 & - & 2 & $3.5 \%$ \\
\hline T. Mentagrophyte \& A.niger & 2 & - & - & 2 & $3.5 \%$ \\
\hline A.niger & - & - & 2 & 2 & $3.5 \%$ \\
\hline Candida albicans & 2 & - & - & 2 & $3.6 \%$ \\
\hline Pencillinum spp. & 1 & - & - & 1 & $1.8 \%$ \\
\hline Fusarium & 1 & - & - & 1 & $1.8 \%$ \\
\hline Total & 30 & 16 & 10 & 56 & $100 \%$ \\
\hline *TP \& O= Tinea Pedis \& Onychomycosis; TP= Tinea Pedis; O= Onychomycosis \\
\hline
\end{tabular}

In squamous hyperkeratotic and intertriginous types of (57.5\%), followed by T. Mentagrophyte (35\%), as Tinea Pedis, T. Rubrum was the most common isolate summarized in table 5 .

Table 5: Fungal species in relation to morphological patterns of T. Pedis

\begin{tabular}{|l|c|c|c|c|}
\hline Causative fungus & Sq. Keratotic & Intertriginous & Intertriginous \& sq. Keratotic & Total \\
\hline T. Rubrum & 14 & 7 & 2 & 23 \\
\hline T. Mentagrophyte & 10 & 4 & 0 & 14 \\
\hline T. Rubrum \& A. Niger & 0 & 1 & 1 & 2 \\
\hline T. Mentagrophyte \& A. Niger & 1 & 0 & 0 & 1 \\
\hline
\end{tabular}


T. Rubrum was most commonly isolated (27\%) from DLSO Onychomycosis pattern. T. Mentagrophyte was equally isolated from cases of DLSO, TDO and mixed DLSO/ TDO (11\% each). Both cases of C. albicans had DLSO morphological type. A.niger was isolated from 2 cases each with DLSO and TDO, as shown in table 6.

Table 6: Fungal species in relation to morphological types of onychomycosis.

\begin{tabular}{|l|c|c|c|c|c|c|}
\hline \multicolumn{1}{|c|}{ Causative fungus } & DLSO & TDO & DLSO \& TDO & SWO & DLSO \& SWO & PSO \\
\hline T. Rubrum & 10 & 3 & 2 & 0 & 0 & 0 \\
\hline T. Mentagrophyte & 4 & 4 & 4 & 1 & 1 & 0 \\
\hline A. Niger & 2 & 2 & 0 & 0 & 0 & 0 \\
\hline C. Albicans & 2 & 0 & 0 & 0 & 0 & 0 \\
\hline Fusarium & 1 & 0 & 0 & 0 & 0 & 0 \\
\hline Pencillinium & 1 & 0 & 0 & 0 & 0 & 0 \\
\hline
\end{tabular}

ome of the culture positive results are shown in fig. 1 and 2.

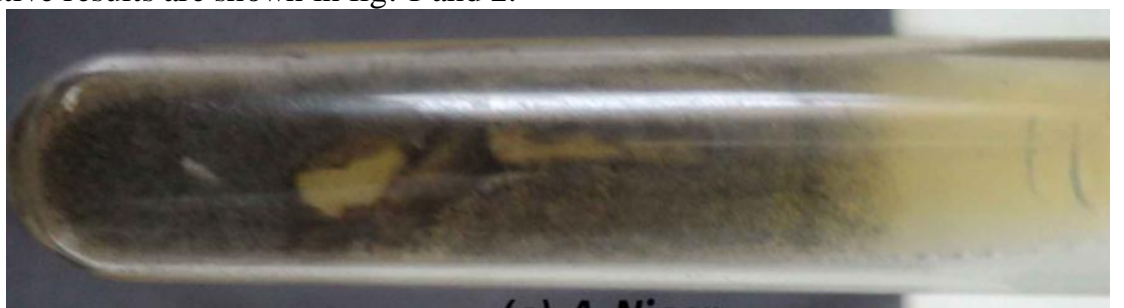

(a) A. Niger

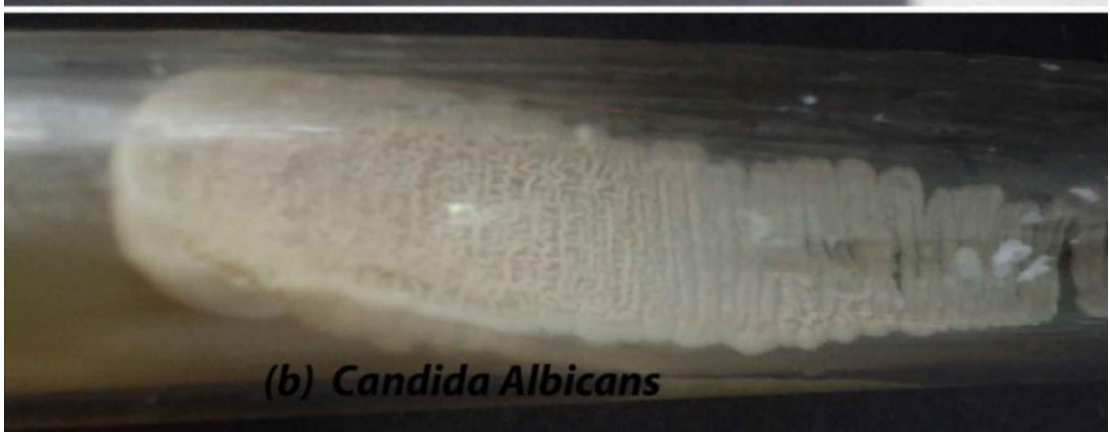

Fig. 1: Growth of A. Niger (a) and Candida (b) on culture.
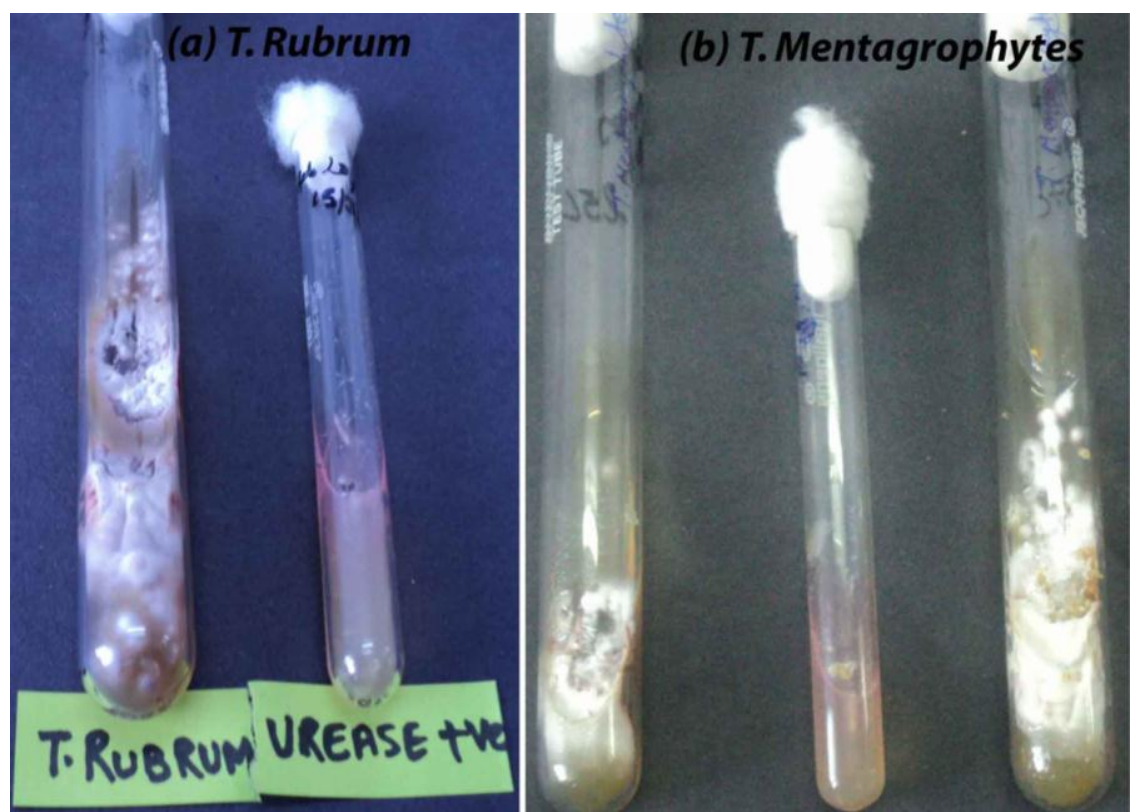

Fig. 2: Growth of T. Rubrum (a) and T. Mentagrophytes (b) on culture. 


\section{Discussion}

In this study, most of the patients were in the age group 21-40 years followed by 41-60 years. Lesser prevalence was found in $\leq 20$ year age group and least prevalence in $\geq 61$ year age group. These findings are consistent with the previous studies from around the world, justifying the statement that Tinea Pedis and Onychomycosis is generally a disease of adults and uncommon in children and elderly. ${ }^{411-20}$

A high prevalence of Tinea Pedis and Onychomycosis in patients above 55 years was found in some previous studies in contrast to ours. ${ }^{14,21,22,23}$ The actual incidence of Tinea Pedis and Onychomycosis may, in fact, be higher in elderly group in our country, because it is very difficult for elderly to seek medical consultation at hospital, as they are dependent upon others to visit the hospital. The low prevalence of Tinea Pedis and Onychomycosis in children is attributed to difference in nail plate structure, a lack of cumulative trauma, lack of occlusive foot wear and increased growth rate of nail plate with subsequent elimination of fungus. ${ }^{24}$

In our study, Tinea Pedis and Onychomycosis were found to be more common in males than females, similar to the observations in various previous studies. $^{2,3,13,17,18,23,25,26,27,28,29}$ In contrast, high incidence in females has been reported in some studies. ${ }^{7}, 20,24,30,31$ Candidial Onychomycosis has been reported to be more common in females in most of the previous studies. ${ }^{2,3,9,20,24}$ In our study also, both of the 2 cases of candidal toe nail Onychomycosis were females. Lesser incidence of Tinea Pedis and Onychomycosis in females reported in literature may not be true in real sense, as the disease being often asymptomatic is not reported as frequently as in males. ${ }^{18,23}$

More number of patients from rural areas than the urban is in proportion to the overall more rural population. Again rural population having farming as main occupation and also chances of more contact with cattle are more liable to get fungal infections.

Occupations which predispose the patient to micro trauma and injuries lead to overall more chances of Tinea Pedis and Onychomycosis. ${ }^{3,8,9,13,32}$ These include farmers, labourers and defence personnel, which comprised respectively $4 \%, 6 \%$ and $13.3 \%$ of all the patients. However, history of obvious trauma preceding the Tinea Pedis and Onychomycosis may not be always given, for example, only 3 patients in our study. In our study, service class being the most common patient group, 35 patients out of 150, may be attributed to more awareness among these, along with easy accessibility to hospital, hence reported more.

Tinea Pedis and Onychomycosis has asymptomatic and indolent course as observed by other workers. ${ }^{11,20,24,26,33}$ In our study also, disease duration of more than 6 months at the time of presentation was found in $88(75.7 \%)$ cases of Tinea Pedis and 86 (95.6\%) cases of Onychomycosis.

Animals may be a source of fungal infections in rural areas, ${ }^{34}$ and in our study also history of contact with cattle and pets was present in $29(19.3 \%)$ patients, although there was no history suggestive of infection in these animals.

Warm and moist environment provided by occlusive footwear promotes growth of fungus and predisposes to Tinea Pedis and Onychomycosis, as observed in previous studies. ${ }^{6,8,21,23,35}$ Similarly, majority of patients in our study had the habit of wearing occlusive shoes and socks.

Distal and lateral subungual Onychomycosis (DLSO) was the commonest type (50\%) encountered in our study, consistent with observations in previous studies. ${ }^{20,29}$ The reason behind this is that the initial site of invasion is usually the free edges, followed by involvement of hyponychium and later nail bed and nail plate at distal and lateral end.

In this study $24.5 \%$ of the cases had total dystrophic Onychomycosis (TDO) and 20\% had both DLSO and TDO in the same patient. Number of cases of TDO was more as compared to some of the previous studies. ${ }^{17,19,29,35}$

Proximal subungual Onychomycosis (PSO), which may be a sign of immunodeficiency, has been very rare variety in previous studies., 9,10,7, 22, 36 It was not present in any of our patients as well.

In our study, 56 out of 150 patients had both Tinea Pedis and toe nail Onychomycosis in the same patient. Tinea Pedis infection preceded the nail involvement in 28 $(50 \%)$ patients and followed it in $16(28.5 \%)$ patients, whereas in $12(21.5 \%)$ patients there was simultaneous involvement of skin and nails. From this we can infer that though transmission of infection between skin and nail is a two way process, yet Tinea Pedis mostly precedes Onychomycosis, because hard difficult-to-dissolve keratinous structure and low nutrient supply of nails do not provide favourable environment for establishment of fungus than the skin, but once established it acts as reservoir for constant source of infection for other parts of the body. ${ }^{8,11,32}$

In our study, dermatophytes were isolated in 46 out of 56 culture positive results $(82.14 \%)$ and most of these were positive within first two weeks of inoculation. These observations show that dermatophytes are the main etiological agents causing Tinea Pedis and Onychomycosis, a fact established by previous studies as well. ${ }^{9,12,27,35}$

Generally, there is always a difficulty in isolating the fungi on culture from the nail material. One of the reasons may be the non-viability of fungal hyphae in the distal portion of the nail plate from where the samples are usually taken. $3,4,8,9,18,20$

The most common isolate obtained in our study was $\mathrm{T}$. Rubrum (50\%), which has been reported as most prevalent pathogen in Tinea Pedis and Onychomycosis in previous studies also. $2,7,9,12,13,17,18,22,23,26,27,35$ T. Rubrum can colonize easily on stratum corneum and hard keratin, so it has greater capacity to infect the skin and nails of the feet than any other dermatophyte. ${ }^{8,22}$

T. Mentagrophyte was the second most common isolate in our study $(32.3 \%)$. Similar observations were made in previous studies also. ${ }^{2,9,13,17,18,29} \mathrm{~T}$. Mentagrophyte has been reported as the most common isolate in some other studies. ${ }^{2,37}$ 
In our study, there were $2(3.5 \%)$ culture positive candida albicans species and these patients had chronic paronychia and nail involvement. The isolation rates of yeasts from the nails have been highly variable. Among all the yeasts, candida species are the most frequently reported ones. ${ }^{3,20,24,35}$

Non-dermatophytes were isolated in 8 cases $(14.2 \%)$ and 6 of them were Aspergillus species, of which 2 cases of Tinea Pedis had mixed culture of A. niger and T. Rubrum. It may be presumed that $\mathrm{T}$. Rubrum was the primary pathogen and moulds secondarily infected the damaged nail. Aspergillus species has also been reported in earlier studies. $^{9,17,19,22,38}$ Other isolates in our study were Penicillinum and Fusarium. Onychomycosis caused by nondermatophytes are often associated with that caused by dermatophytes. Non-dermatophyte Onychomycosis is usually confined to toe nails and often asymptomatic, which may be the reason for its low incidence due to under reporting. ${ }^{32}$

In our study T. Rubrum was the most common isolate in squamous hyperkeratotic and intertriginous types of Tinea Pedis, followed by T. Mentagrophyte in both groups, similar to previous reported studies. ${ }^{2,28}$

T. Rubrum was found to be the commonest cause of DLSO and TDO in our study. DLSO is the commonest type of nail dystrophy and is usually caused by T. Rubrum. ${ }^{9,17}$

\section{Conflict of Interest: None.}

\section{Financial Support: None.}

\section{Conclusion}

Although all the 150 clinically diagnosed cases with Tinea Pedis and Toe nail Onychomycosis alone or in combination pattern, were selected for the study after establishing $\mathrm{KOH}$ positivity for fungal elements in all the cases, yet the culture results for fungal growth correlated in $56(37.3 \%$ ) patients only. Among these $53.3 \%$ (30 out of 56) patients with both Tinea Pedis and Onychomycosis, $26.7 \%$ (16 out of 60 ) with Tinea Pedis only and 29.4\% (10 out of 34) with Onychomycosis only were culture positive. The most frequent growth obtained was that of T. Rubrum which was isolated in 28 out of $56(50 \%)$ patients, followed by $\mathrm{T}$. Mentagrophyte, isolated in $18(32.1 \%)$ cases. Nail plate biopsy followed by periodic acid-Schiff staining should be the most accurate method for diagnosing fungal elements in Onychomycosis, but this facility was not available in our laboratory.

\section{References}

1. Leyden JJ, Kligman AM. Inter-digital Athlete's Foot. Arch Dermatol 1978;114:1466-1472.

2. Clayton YM. Clinical and mycological diagnostic aspects of Onychomycosis and dermatomycoses. Clin Exp Dermatol 1992; 17: 37-40.

3. Haneke E. Fungal infections of the nail. Semin Dermatol 1991; 10: 41 .
4. Gupta AK, Sibbald RG, Lynde RG, et al. Onychomycosis in children: prevalence and treatment strategies. J Am Acad Dermatol 1997;36:395-402.

5. Jain S, Seghal VN. Onychomycosis: an epidemio-etiologic perspective. Int J Dermatol 2000; 39: 100-103.Asadi MA, Dehghani R, Sharif MA. Epidemiologic study of Onychomycosis and Tinea Pedis in Kashan, Iran. Jundishapur J Microbiol 2009;2:61-64.

6. Gupta AK, Jain HC, Lynde CW, et al. Prevalence and epidemiology of Onychomycosis. J Am Acad Dermatol 2000;43:244-248.

7. English MP. Nail and fungi. Br J Dermat 1976; 94: 697

8. Andre J, Achten G. Onychomycosis. Int J Dermatol 1987; 26: 481-490.

9. Elewski BE. Onychomycosis: Pathogenesis, Diagnosis, and Management. Clinical Micro 1998; 11: 415-429.

10. Zaias N. Onychomycosis. Dermatol Clin 1985; 3: 445-460.

11. Kam KM, Au WF, Wong PY, et al. Onychomycosis in Hong Kong. Int J Dermatol 1997; 36: 757-761.

12. Perea S, Ramos MJ, Garau M, et al. Prevalence and risk factors of Tinea Unguium and Tinea Pedis in the General population in Spain. J Clin Microbiol 2000; 38: 3226-3230.

13. Djeridane A, Djeridane Y, Khodje AA. Epidemiology and aetiological study on Tinea Pedis and Onychomycosis. Mycoses 2006:49:190-196.

14. Adhikari L, Gupta AD, Pal R, et al. Clinico-etiologic correlates of Onychomycosis in Sikkim. Indian J Pathol Microbiol 2009;52:194-197.

15. Reddy BSN, Singh G, Sharma BN. Onychomycosis and nail dystrophy. Indian J Dermatol 1977;23:2.

16. Ramesh V, Singh R, Reddy BSN, et al. Clinico-mycological study of Onychomycosis. Indian J Dermatol Venereol Leprol 1982;48:145-150.

17. Banerjee U, Sethi M, Pasricha JS. Study of Onychomycosis in India. Mycoses 1989;33:411-415.

18. Sujatha V, Grover S, Dash K, et al. A clinico-mycological evaluation of Onychomycosis. Indian J Dermatol Venereol Leprol 2000;66:238-240.

19. Jesudanam TM, Rao RGR, Lakshmi DJ. Onychomycosis: a significant medical problem. Indian J Dermatol venereal leprol 2002;68:326-329.

20. Roberts DT. Prevalence of Dermatophyte Onychomycosis in the United Kingdom: results of an omnibus survey. $\mathrm{Br} \mathrm{J}$ Dermatol 1992; 126: 23-27.

21. Hay RJ. Fungal skin infections. Arch Dis Child 1992; 67: 1065-1067.

22. Kaur R, Kashyap B, Makkar R. Evaluation of clinicomycological aspects of Onychomycosis. Indian $J$ Dermatol 2008;53:174-178.

23. Bokhari MA, Hussain I, Jahangir M, et al. Onychomycosis in Lahore, Pakistan. Int J Dermatol 1999;38:591-595.

24. Scher RK. Onychomycosis: a significant medical disorder. $J$ Am Acad Dermatol 1996;35:2-5.

25. Roseeuw D. Achilles foot screening project: preliminary results of patients screened by dermatologists. $J$ Eur Acad Dermatol Venerol 1999;12:6-9.

26. Vinod S, Grover S, Dash K, et al. A clinico-mycological evaluation of Onychomycosis. Indian J Dermatol Venereol Leprol 2000;66:238-240.

27. Cheng S, Chong L. A Prospective Epidemiological study of Tinea Pedis and Onychomycosis in Hong Kong. Chinese Med $J$ 2002;195:860-865.

28. Garg A, Venkatesh V, Singh M et al. Onychomycosis in central India: a clinic-etiologic correlation. Int J of Dermatol 2004;43:498-502.

29. Velez A, Linares MJ, Fenandez-Roldan JC, et al. Study of Onychomycosis in Cor doba, Spain: prevailing fungi and pattern of infection. Mycopathologia 1997;137:1-8. 
30. Lopes JO, Alves SH. A ten year survey of Onychomycosis in the central region of Rio Grande, Brazil. Rev Inst Med Trolp Sao Paulo 1999;41:147-149.

31. Walshe MM, English MP. Fungi in nails. Br J Dermatol 1966;98:198-208.

32. Hay RJ. Chronic Dermatophyte Infections - I. Clinical mycological features. Br J Dermatol 1982;106:1-5.

33. Georg LM. Observations on rural and urban ringworm. Invest Dermatol 1956;27:325-326.

34. Grover S. Clinico-mycological evaluation of Onychomycosis at Banglore and Jorhat. Indian J Dermatol Venereal Leprol 2003;69:286-289.
35. Dompmartin D, Dompmartin A, Deluol AM, et al. Onychomycosis and AIDS: clinical and laboratory findings in 62 patients. Int J Dermatol 1990;29:337-339.

36. Merlin K, Kilkenny M, Plunkett A, et al. The prevalence of common skin conditions in Australian school students: 4 Tinea Pedis. Br J 1999;140:897-901.

37. Dermatol Srinivas CR, Ramani A, Kumari GR, et al. Moulds in Onychomycosis. Int J Dermtol 1993;32:877-878.

How to cite this article: Bhatti R, Rather PA, Dogra D, Gupta V, Mahajan B, Clinico-Mycological study of tinea pedis and toe nail onychomycosis: A multi-center study from Jammu. Indian J Clin Exp Dermatol 2019;5(1):54-60 